\title{
Fast quantitative susceptibility mapping using 3D EPI and total generalized variation
}

Citation for published version (APA):

Langkammer, C., Bredies, K., Poser, B. A., Barth, M., Reishofer, G., Fan, A. P., Bilgic, B., Fazekas, F., Mainero, C., \& Ropele, S. (2015). Fast quantitative susceptibility mapping using 3D EPI and total generalized variation. Neuroimage, 111, 622-630. https://doi.org/10.1016/j.neuroimage.2015.02.041

Document status and date:

Published: 01/01/2015

DOI:

10.1016/j.neuroimage.2015.02.041

Document Version:

Publisher's PDF, also known as Version of record

Document license:

Taverne

Please check the document version of this publication:

- A submitted manuscript is the version of the article upon submission and before peer-review. There can be important differences between the submitted version and the official published version of record.

People interested in the research are advised to contact the author for the final version of the publication, or visit the DOI to the publisher's website.

- The final author version and the galley proof are versions of the publication after peer review.

- The final published version features the final layout of the paper including the volume, issue and page numbers.

Link to publication

\footnotetext{
General rights rights.

- You may freely distribute the URL identifying the publication in the public portal. please follow below link for the End User Agreement:

www.umlib.nl/taverne-license

Take down policy

If you believe that this document breaches copyright please contact us at:

repository@maastrichtuniversity.nl

providing details and we will investigate your claim.
}

Copyright and moral rights for the publications made accessible in the public portal are retained by the authors and/or other copyright owners and it is a condition of accessing publications that users recognise and abide by the legal requirements associated with these

- Users may download and print one copy of any publication from the public portal for the purpose of private study or research.

- You may not further distribute the material or use it for any profit-making activity or commercial gain

If the publication is distributed under the terms of Article $25 \mathrm{fa}$ of the Dutch Copyright Act, indicated by the "Taverne" license above, 


\title{
RF peak power reduction in CAIPIRINHA excitation by interslice phase optimization
}

\author{
Alessandro Sbrizzi ${ }^{a *}$, Benedikt A. Poser ${ }^{b}$, Desmond H. Y. Tse ${ }^{b, c}$, \\ Hans Hoogduin ${ }^{a}$, Peter R. Luijten ${ }^{a}$ and Cornelis A. T. van den Berg ${ }^{a}$
}

\begin{abstract}
The purpose of this work was to show that the overall peak power of RF pulses for CAIPIRINHA excitation can be substantially reduced by applying interslice phase relaxation. The optimal phases are scan dependent and can be quickly calculated by the proposed method.

The multi-band RF pulse design is implemented as the minimization of a linear objective function with quadratic constraints. The interslice phase is considered to be a variable for optimization. In the case of a phase cycling scheme (CAIPIRINHA), the peak power is considered over all pulses.

The computation time (about $1 \mathrm{~s}$ ) is compatible with online RF pulse design. It is shown that the optimal interslice phases depend on the CAIPIRINHA scheme used and that RF peak power is reduced when the CAIPIRINHA phase cycling is taken into account in the optimization.

The proposed method is extremely fast and results in RF pulses with low peak power for CAIPIRINHA excitation. The MATLAB implementation is given in the appendix; it allows for online determination of scan-dependent phase parameters. Furthermore, the method can be easily extended to pTx shimming systems in the context of multi-slice excitations, and this possibility is included in the software. Copyright $\odot 2015$ John Wiley \& Sons, Ltd.
\end{abstract}

Keywords: RF pulse design; CAIPIRINHA; multi-band; RF peak power; interslice phase relaxation; simultaneous multi-slice; active-set; linear program

\section{INTRODUCTION}

The quest for faster MRI scans has recently led to the revival of multi-band imaging (1-3), where several slices are simultaneously excited while a 2D field of view (FOV) is acquired. The resulting image can be unfolded by exploiting the sensitivity profiles of the receive coils (4-6). The controlled-aliasing-in-parallel-imaging-results-in-higher-acceleration (CAIPIRINHA) method (7) has increased the efficiency of the multi-band techniques. This approach relies on adding a phase off-set pattern for readout lines of 2D acquisitions. The effective linear phase modulation obtained along the phase encoding direction results in a shift of the image. Images belonging to different slices do not collapse on top of each other, thus are easier to unfold (lower $g$-factor penalty) $(7,8)$. The phase cycling effect can be obtained in two different ways: by a gradient blip (9) or by a slice-dependent phase term in the RF excitation (7). The first solution requires fast control of the gradients and accurate eddy current compensation. In addition, only regular phase schemes are obtainable, in which the slice shift is proportional to the slice distance from the isocenter.

The second method employs RF excitations to achieve the slice shifts, specifically by cycling through a set of multi-band RF pulses that contain the desired slice-specific phase cycling pattern. This approach is more flexible than using gradient blips, as any arbitrary slice shift can be produced for each slice, independent of the relative slice position. However, RF-induced phase cycling can not be obtained in an EPI scan, which is an important tool for, among others, fMRI and DTI studies. The two methods share the need for multi-band pulses, which in the case of simple complex summation of the $N$ corresponding single-band pulses will have a factor of $N$ higher transmitter voltage $\left(N^{2}\right.$ higher peak power) $(2,10)$. This sets constraints on the examination time and results in pulses with a narrower frequency bandwidth.

To overcome excessive RF peak power, the so-called VERSE technique (11) can be applied. In addition, (12) shows that by increasing the duration of the multi-band pulse and time-shifting the different frequency bands, the peak voltage can be reduced. A recently introduced method to effectively reduce the RF power is the so-called power-independent-of-number-of-slices (PINS) technique (13), which relies on a periodical slice excitation to uniformly cover the volume of interest. Its application is not possible when the excitation is reduced to a portion of the FOV, and it does not allow RF-induced phase cycling.

\footnotetext{
* Correspondence to: Alessandro Sbrizzi, Imaging Division, University Medical Center Utrecht, Utrecht, The Netherlands.

E-mail: a.sbrizzi@umcutrecht.nl

a A. Sbrizzi, H. Hoogduin, P. R. Luijten, C. A. T. Berg

Imaging Division, University Medical Center Utrecht, Utrecht, The Netherlands

b B. A. Poser, D. H. Y. Tse

Faculty of Psychology and Neuroscience, Maastricht University, Maastricht, The Netherlands

C D. H. Y. Tse

Department of Radiology, Maastricht University Medical Center, Maastricht, The Netherlands
}

Abbreviations used: CAIPIRINHA, controlled aliasing in parallel imaging results in higher acceleration; DTI, diffusion tensor imaging; EPI, echo-planar imaging; fMRl, functional MRI; FOV, field of view; MB, multi-band; $p T x$, parallel transmit excitation; $R x$, receive; $T X$, transmit; VERSE, variable-rate selective excitation. 
Moreover, the waveforms become longer and thus more sensitive to off-resonance effects, as mentioned above.

An approach to mitigate the peak voltage (or peak power) problem in regular multi-band pulses was proposed for the first time in (14) and recently extended in (15): through relaxation of the phase of the target magnetization profile, it is possible to design multi-band RF pulses with lower peak amplitude. To achieve this, a minimization problem is solved that considers the phase of each slice as a variable of the optimization. The optimal interslice phases for $N \leq 16$ have been tabulated in (15).

In this work, we extend the principle of interslice phase relaxation to multi-band RF pulses required for CAIPIRINHA imaging. We will show that the interslice phase optimization depends upon the particular phase cycling scheme required for the signal acquisitions: pulses that excite different phase profiles (required for the image shift) show different interference patterns. The CAIPIRINHA cycling scheme and thus the corresponding optimal phase relaxation values are scan dependent. Tabulating all possible combinations as presented for the unmodulated MB excitation in (15) would be infeasible, since not only the number of slices, but also the CAIPIRINHA factor and the specific phase cycling patterns influence the optimal phases. Furthermore, when pTx systems are employed, the patient-specific amplitude and phase of each transmit channel for each slice have to be taken into account. It follows that the phase optimization has to be performed online on the scanner.

In this paper, we demonstrate an efficient algorithm that returns the optimal phases in about $1 \mathrm{~s}$ on a standard desktop PC. Control of the RF peak power over all RF pulses of a CAIPIRI$\mathrm{NHA}$ excitation is quickly obtained. For comparison, the computation time required by the method based on (15) for 1000 restarts is of the order of minutes.

The mathematical derivation of the design process is illustrated in the theory section, with more details in the appendix. Three numerical experiments are presented, in which speed, robustness and performance of the new approach are evaluated. It will be shown that (a) control and minimization of the RF peak power over a whole CAIPIRINHA cycling scheme are necessary and are obtained by the proposed method, (b) the achieved RF peak amplitude values are close to the theoretical minimum value of $\sqrt{N}$ (where $N$ is the number of slices) (15) and (c) the computation time allows for online, scan-dependent implementation of the interslice phase optimization. Although not strictly necessary for the validation of the new algorithm, a scanner experiment is performed to prove the practical feasibility of the obtained pulses. This is demonstrated in a phantom at $7 \mathrm{~T} \mathrm{MRI}$

The MATLAB code is provided in the appendix.

\section{THEORY}

Given a single-band RF waveform $b(t)$ and the corresponding slice selective gradient value, $G$, the RF waveform $f$ that excites $N$ slices at locations $z_{n}(n=1, \ldots, N)$ is given by

$$
f(t)=\sum_{n=1}^{N} b(t) \mathrm{e}^{\mathrm{i} \gamma G t z_{n}}
$$

The constructive interference of the complex exponential terms $\mathrm{e}^{\mathrm{i} \gamma \mathrm{Gt} z_{n}}$ quickly leads to high peak power (15).
To overcome this problem, Hennig (14) and Wong (15) suggest relaxing the phase of each slice, namely, to add phases $\phi_{n}$ $(n=1, \ldots, N)$ to the complex exponentials. The resulting RF waveform becomes

$$
f(t, \vec{\phi})=b(t) \sum_{n=1}^{N} \mathrm{e}^{\mathrm{i}\left(\gamma G t z_{n}+\phi_{n}\right)}
$$

where $\vec{\phi} \equiv\left[\phi_{1}, \ldots, \phi_{N}\right]$. A proper choice of $\vec{\phi}$ leads to a favorable interference of the complex exponential terms, which results in a drastic reduction of the peak power of $f$. The terms in the sum represent a mode beating that can result in destructive interference by optimizing $\vec{\phi}$. This kind of problem is relevant in other applications, for instance in optics. Its theoretical minimum is $\sqrt{N}(15)$, but this is not necessarily attainable (see also the discussion about the interference of various spontaneous atomic emitters in (16)).

\section{Phase cycling schemes}

Recent developments in multi-band imaging include the socalled CAIPIRINHA technique (7). A lower $g$-factor penalty can be obtained when images from different slices are superimposed with an off-set in the phase encoding direction. One way to achieve this off-set is to cycle the RF excitation over a phase cycling scheme $\Psi=\left(\psi_{p, n}\right)$, where $\psi_{p, n} \in[0,2 \pi)$ denotes the phase of the $n$th slice obtained from the $p^{\text {th }}$ RF.

For example,

$$
\Psi_{\mathrm{a}}=\left(\begin{array}{lllll}
0 & 0 & 0 & 0 & 0 \\
\pi & 0 & \pi & 0 & 0
\end{array}\right)
$$

implies that two five-band excitation pulses are alternated. The first RF pulse leaves the phases unchanged and the second one applies a $180^{\circ}$ phase to the first and the third slice. By alternating the two RF waveforms for every $k$-space line, the resulting folded image will exhibit a shift by FOV/2 for Slices 1 and 3 with respect to Slices 2, 4 and 5 . Note that this asymmetric cycling scheme might be required in cases when only the first three slices are affected by high g-factor values, in which case no shift is needed for Slice 5 . Asymmetric phase cycling schemes can also result from algorithmic design of CAIPIRINHA sequences, as shown in (17). In a similar way, the phase cycling scheme

$$
\Psi_{b}=\left(\begin{array}{cccc}
0 & 0 & 0 & 0 \\
0 & \frac{\pi}{2} & \pi & \frac{3 \pi}{2} \\
0 & \pi & 0 & \pi \\
0 & \frac{3 \pi}{2} & \pi & \frac{\pi}{2}
\end{array}\right)
$$

requires the alternation of four RF pulses, and the resulting folded image will exhibit shifts by 0, FOV/4, FOV/2 and 3FOV/4 for Slices $1,2,3$ and 4 , respectively.

A phase cycling scheme has a direct effect on the shape of the corresponding RF waveform, and thus on the peak amplitude. Therefore, an optimal phase relaxation scheme for a standard (not CAIPIRINHA) multi-band excitation could still give rise to a high peak amplitude when the phase of each slice is cycled according to a given $\Psi$. To show this, suppose the excitation scheme given by $\Psi_{a}$ is required. Applying the phases reported in (15) for a two-slice excitation, one obtains 
the pulses shown in Fig. 1. Note that, while the optimal phases are effective in reducing the peak power of the first pulse, they are unable to lower it for the second one. The overall peak value (that is, the maximum over Pulses 1 and 2) is just $10 \%$ lower than the peak value for the standard, nonoptimized pulse.

The previous example shows the need for scan-dependent interslice phase optimization, which is closely connected to the CAIPIRINHA phase settings $\Psi$.

The optimization procedure employed in (15) does not include the CAIPIRINHA cycling. In addition, it could require a large number of restarts, as reported in (15), where 5000 restarts are used. This makes the online implementation unfeasible. For these reasons, we propose an efficient implementation of the CAIPIRINHA-dependent phase optimization.

\section{Optimization}

We first derive a model for the problem. Given a pulse $b$, slice selective gradient waveform $G$, slice positions $z_{n}$ and a CAIPIRINHA scheme $\Psi$, the $p$ th pulse of the excitation scheme is given by

$$
f^{p}(t, \vec{\phi})=\sum_{n=1}^{N} b(t) \mathrm{e}^{\mathrm{i}\left(\gamma G t z_{n}+\psi_{p, n}+\phi_{n}\right)} .
$$

The CAIPIRINHA interslice optimization problem becomes that of finding the optimal phase vector $\phi^{*}$ that minimizes the peak RF over all pulses.

In compact mathematical notation,

$$
\bar{\phi}^{*}=\arg \min _{\bar{\phi}} \max _{t, p}\left|f_{p}(t, \vec{\phi})\right| \text {. }
$$

The problem given in Equation [6] is not convex, and the objective is not differentiable with respect to $\phi$. This explains why many restarts are needed to obtain a good solution. In addition, the non-differentiability implies that the gradient of the function is not explicitly obtained, resulting in slower convergence behavior. In the appendix, we derive an equivalent expression for the peak minimization problem that is differentiable and shows fewer problems with respect to local minima.
The problem can eventually be cast as the minimization of a linear objective with quadratic equality and inequality constraints:

$$
\begin{aligned}
& \text { minimize } \boldsymbol{c}^{\top} \boldsymbol{y} \\
& \text { such that } \boldsymbol{y}^{\top} \mathbb{Q}_{h} \boldsymbol{y} \leq 0, \quad \forall h \\
& \boldsymbol{y}^{\top} \mathbb{P}_{n} \boldsymbol{y}-1=0, \quad n=1, \ldots, N .
\end{aligned}
$$

The gradients of the objective and constraint functions with respect to $\boldsymbol{y}$ are easily derived (note that $Q_{h}$ and $P_{n}$ are symmetric matrices):

$$
\begin{gathered}
\nabla \boldsymbol{c}^{\top} \boldsymbol{y}=\boldsymbol{c}^{\top} \\
\nabla \boldsymbol{y}^{\top} \mathbb{Q}_{h} \boldsymbol{y}=2 \boldsymbol{y}^{\top} \mathbb{Q}_{h} \\
\nabla\left(\boldsymbol{y}^{\top} \mathbb{P}_{n} \boldsymbol{y}-1\right)=2 \boldsymbol{y}^{\top} \mathbb{P}_{n} .
\end{gathered}
$$

We solve the problem using an active-set algorithm. For this work, we employed the built-in MATLAB function fmincon from the Optimization toolbox with the gradients supplied as shown above.

The initial guess is randomly generated. The algorithm is repeated a few times, each time with a different starting value. The smallest obtained objective value and the corresponding phase profiles are finally retained as the optimal solution. In practice, we found that five different initial guesses are sufficient to achieve a minimum very close to $\sqrt{N}$, thus the algorithm is restarted five times, independently of the number of slices.

The MATLAB code is presented in Appendix B. The inline comments provide an explanation of the main steps.

\section{METHODS}

The performance of the peak minimization algorithm will be tested in three different ways. They are listed below. For all experiments, we consider a truncated sinc pulse with two side lobes discretized on a $5.0 \mu$ s temporal resolution. The pulse duration is $2.560 \mathrm{~ms}$ and the slice selective gradient value $G$ is $12.0 \mathrm{mT} / \mathrm{m}$, resulting in a $3 \mathrm{~mm}$ slice thickness.
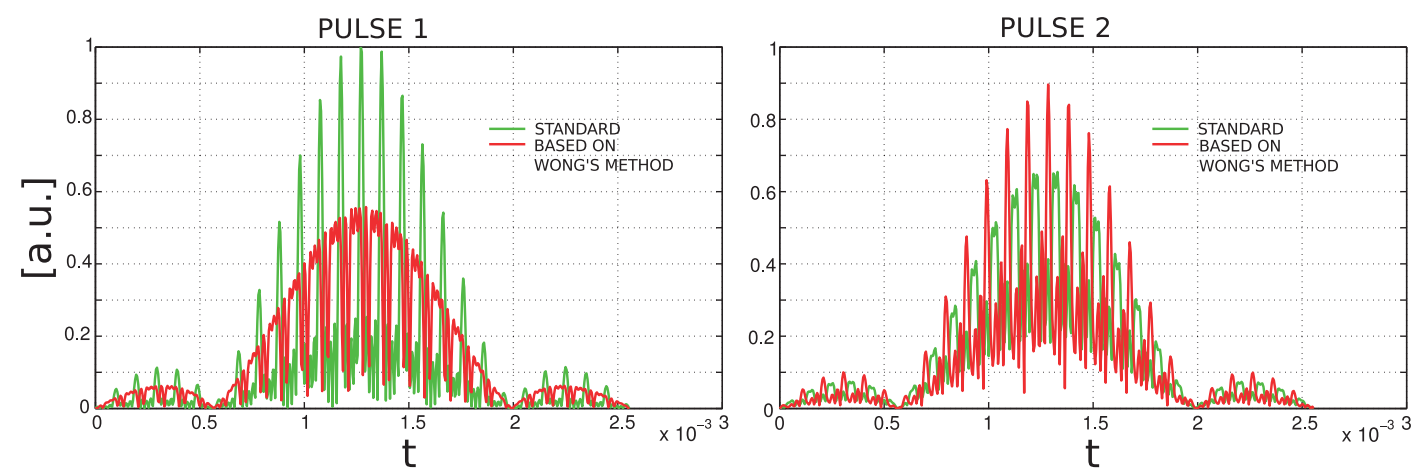

Figure 1. The amplitude of two pulses for the CAIPIRINHA phase cycling scheme $\Psi_{a}=[0,0,0,0,0 ; \pi, 0, \pi, 0,0]$. The standard setup does not include interslice phase relaxation. Note the high peak values of Pulse 1 and Pulse 2 for the standard (green) and Wong's method based (red) pulses, respectively. 


\section{Experiment 1. Online peak minimization for multi-band RF excitation}

The aim of the first experiment is to test the performance of the proposed method in the case of a multi-band excitation for $N=$ $2, \ldots, 16$ (as in the previous equations, $N$ denotes the number of slices). The interslice distance (distance between slice centers) is $\frac{F O V}{N-1}$. There is no CAIPIRINHA cycling. This means that $\Psi$ is a zero row vector: $\Psi=\left[\begin{array}{lll}0 & 0 & \ldots\end{array}\right]$. The achieved peak amplitude for each configuration will be compared with the one achieved for a standard multi-band pulse (no phase relaxation) and the one reported in (15).

The optimal phase relaxation problem is not convex, thus it may converge to local minima. For the case $N=6$ slices, a convergence comparison between the two phase relaxation methods is presented. Both algorithms are restarted 1000 times, each time with common different starting values, and the achieved peak values compared.

\section{Experiment 2. RF peak reduction in joint multi-band/CAIPIRINHA phase cycling schemes}

This experiment illustrates the ability to reduce the peak power for a CAIPIRINHA RF excitation scheme. Consider the previously introduced phase scheme

$$
\Psi_{\mathrm{a}}=\left(\begin{array}{lllll}
0 & 0 & 0 & 0 & 0 \\
\pi & 0 & \pi & 0 & 0
\end{array}\right) .
$$

As we have shown in the methods section (Fig. 1), the CAIPIRINHA phase settings can have a major effect on the RF peak values. We apply the proposed algorithm to the same CAIPIRINHA excitation scheme and we compare the obtained peak values.

To show the reduction of peak power in another CAIPIRINHA setting, we choose the phase cycling $\Psi_{c}$ given by

$$
\Psi_{\mathrm{c}}=\left(\begin{array}{cccccc}
0 & 0 & 0 & 0 & 0 & 0 \\
0 & \pi & 0 & \frac{3 \pi}{2} & 0 & \frac{\pi}{2} \\
0 & 0 & 0 & \pi & 0 & \pi \\
0 & \pi & 0 & \frac{\pi}{2} & 0 & \frac{3 \pi}{2}
\end{array}\right)
$$

and compare the four obtained pulses with the standard ones (no phase relaxation).
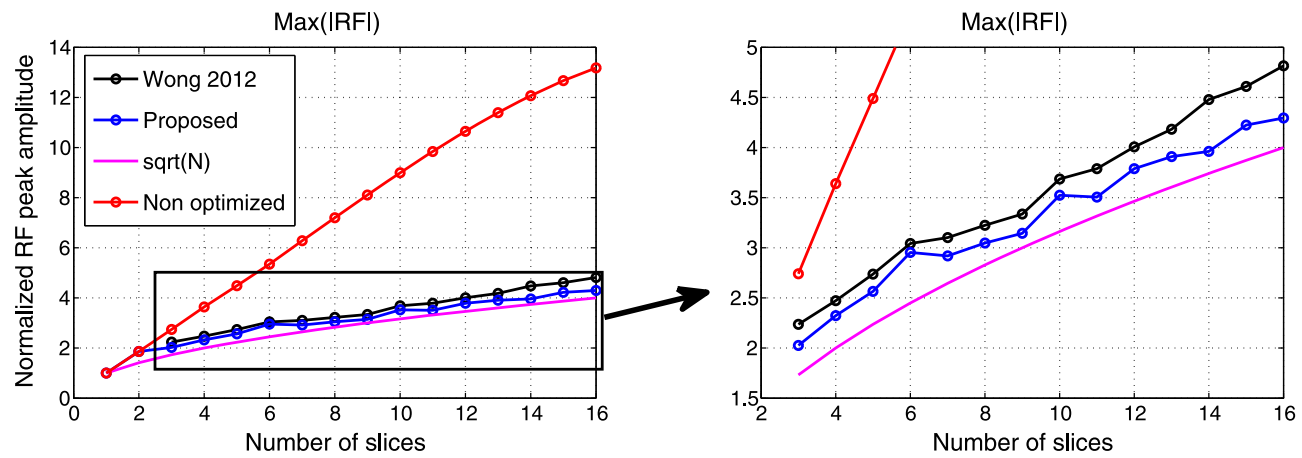

Figure 2. Experiment 1: peak amplitudes for multi-band configurations corresponding to $N=2, \ldots, 16$. Values are normalized with respect to the peak amplitude of $N=1$. For reference, the theoretical minimum value $\sqrt{N}$ is also shown. Note that, concerning the achieved RF peak, the interslice phase relaxation pulses outperform the non-optimized ones. The proposed approach is very close to the theoretical minimum.

\section{Experiment 3. Scanner validation}

To test the practical implementation of the obtained pulses, we perform an experiment on a Siemens MAGNETOM 7T scanner for the CAIPIRINHA scheme given by $\Psi_{a}$, that is, five slices excitation, CAIPIRINHA factor 2. Data were acquired with a 32-channel volume Tx/Rx birdcage head coil (7T Volume T/R, Nova Medical, Wilmington, MA, USA). The nominal slice thickness is $5 \mathrm{~mm}$, and the distance between the centers of the slices is $15 \mathrm{~mm}$. To fully investigate the accuracy of the obtained excitations, we choose a $3 \mathrm{D}$ protocol encompassing all excited slices. In this way, the amplitude and phase of the slice profiles as well as in-plane FOV/2 shifts can be carefully observed.

The matrix size is $64 \times 128 \times 256$, and the resolution in the $z$ direction is $0.3 \mathrm{~mm}$. Additional scan parameters are $T_{\mathrm{E}}=8 \mathrm{~ms}, T_{\mathrm{R}}=$ $15 \mathrm{~ms}$ and nominal flip angle $=20^{\circ}$. To check the slice profiles and the interslice phases obtained from all pulses, we first use

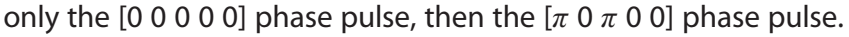
Finally, the experiment is executed by alternating both pulses. An oil-filled ball phantom with $160 \mathrm{~mm}$ diameter is scanned, and the whole experiment is repeated twice: once for the standard pulses and once for the proposed pulses as calculated in Experiment 2 (see the previous subsection).

\section{RESULTS}

\section{Experiment 1. Multi-band RF excitation}

The values of the achieved peak amplitude for $N=1, \ldots, 16$ are plotted in Fig. 2. The theoretical minimum peak amplitude value of $\sqrt{N}$ is plotted as a reference. In the same figure, the values obtained by the standard, non-optimized method and by ref. (15) are also reported. The values from the standard method increase in an approximately linear way, achieving 7.20 for $N=8$ and 13.18 for $N=16$. Note the drastic reduction in peak amplitude obtained by the phase relaxation approach.

The proposed method achieves lower peak amplitude values for each multi-band excitation experiment and it approaches the $\sqrt{N}$ curve. The computation time is of the order of seconds and never longer than $2 \mathrm{~s}$, depending on $N$ (large values of $N$ require more time).

The results from the convergence study are shown in Fig. 3: the histograms of the peak values obtained over 1000 restarts of both methods for $N=6$ are plotted. The peak values achieved by the proposed method never exceed the lowest value by more than $10 \% .50 \%$ of the obtained phase relaxation vectors achieve 

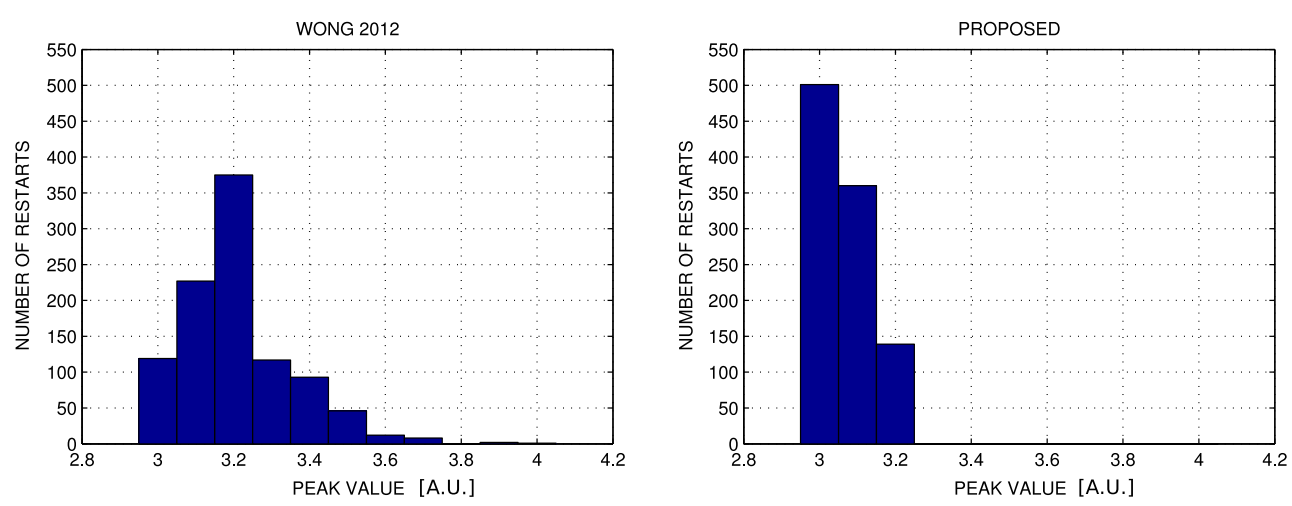

Figure 3. Experiment 1: convergence performance of Wong 2012 (left) and the proposed method (right). 1000 runs of both algorithms are performed, each for different starting values. The distribution of the finally obtained peak values are shown in the histogram plots. Note that $50 \%$ of the restarts from the proposed method converge to the lowest peak values, in contrast with just $12 \%$ from the Wong's method.

the lowest peak value. In contrast, the Wong method has a less robust convergence behavior: the achieved peak values are spread over a larger interval and the lowest value is obtained only $12 \%$ of the times.

\section{Experiment 2. RF peak reduction in joint multi-band/CAIPIRINHA phase cycling schemes}

The RF pulses for the $\Psi_{\mathrm{a}}$ scheme are plotted in Fig. 4. The pulses are scaled with respect to the peak value of standard pulses; see also Fig. 1. The peak value of the proposed method is 0.63 , which is a clear reduction with respect to the pulses designed without taking into account the CAIPIRINHA scheme. Note that both pulses now have a low peak value, illustrating the desired effect of overall RF peak reduction.

A major reduction in peak power is also obtained for the scheme $\Psi_{c}$ (see Fig. 5). As already noticed in the previous case, the waveforms obtained by the proposed method exhibit a more uniform profile, achieving similar peak values for all four pulses. To appreciate the RF peak reduction, the waveforms for the standard (no phase relaxation) pulses are also plotted: they exhibit larger profile variations, and larger overall peak values.
From these two tests it is clear that the CAIPIRINHA scheme has to be taken into account for optimal interslice phase determination.

The total computation time required by our method is $1.1 \mathrm{~s}$ and $1.2 \mathrm{~s}$ for the configurations given by $\Psi_{\mathrm{a}}$ and $\Psi_{\mathrm{c}}$, respectively.

\section{Experiment 3. Scanner validation}

The data from the 3D scans is presented in the following ways.

- The phase of the images obtained with the $\left[\begin{array}{lllll}0 & 0 & 0 & 0 & 0\end{array}\right]$ excitation is subtracted from that of the images with the $\left[\begin{array}{llll}\pi & 0 & \pi & 0\end{array}\right]$ phase excitation. The aim is to check whether the phase difference between the two excitations and the slice profiles are the desired ones. The amplitude and phase of the profile taken along the $z$ direction are shown in Fig. 6. To avoid phase jumps around $\pm 180^{\circ}$, the phase profiles are shifted by $90^{\circ}$. Note that both amplitude and phases are accurately retained.

- The results from an experiment with alternating pulses are shown in Fig. 7. As expected, the images corresponding to
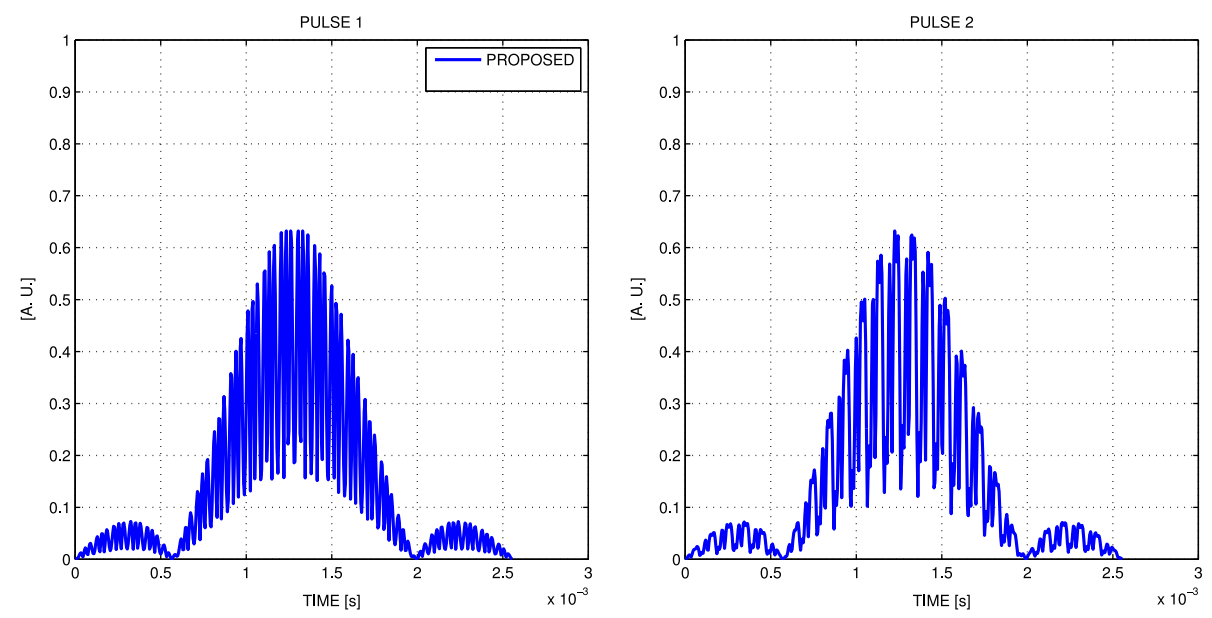

Figure 4. Experiment 2: pulses obtained by the proposed interslice phase relaxation method for the five-band CAIPIRINHA excitation given by $\Psi_{\mathrm{a}}$. The proposed method is able to control the overall peak power by taking into account the CAIPIRINHA scheme during the optimization. The peak value of 0.63 is far below than the one obtained without accounting for CAIPIRINHA phase cycling. The pulses are scaled such that the peak value of the standard (no phase relaxation) pulse is 1 . 

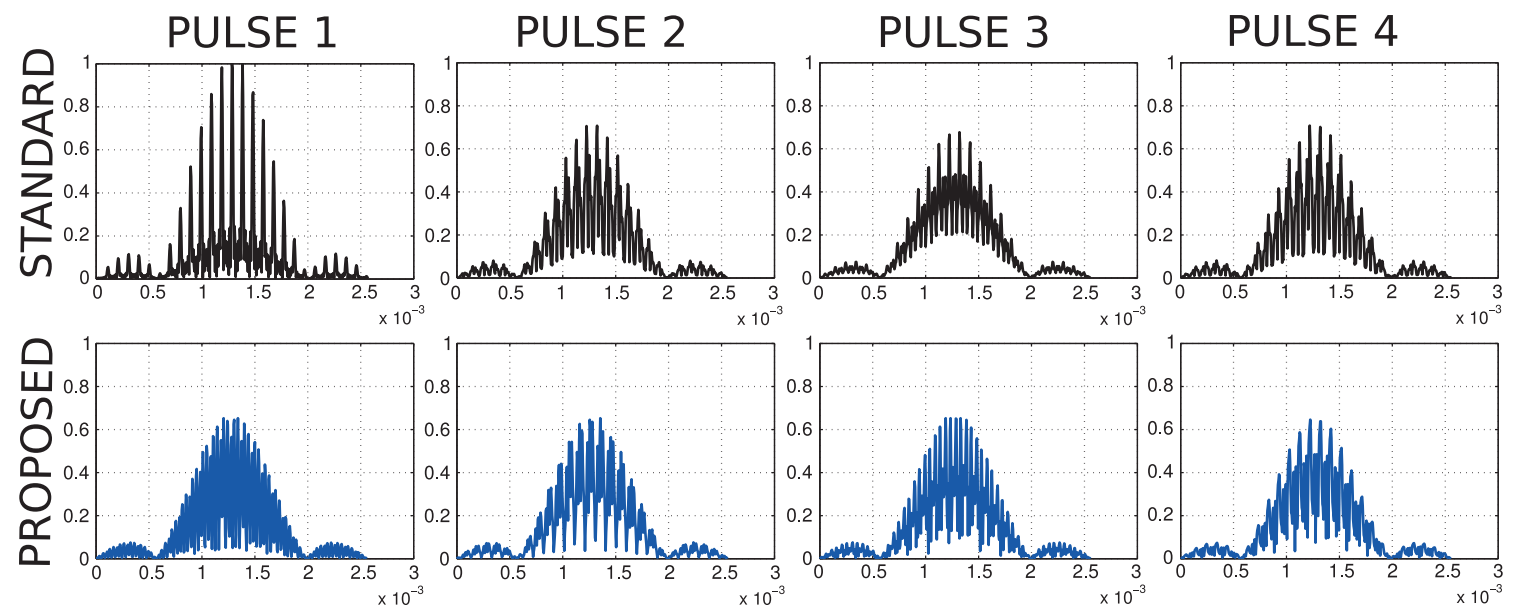

Figure 5. Experiment 2: pulses for the CAIPIRINHA excitation given by $\Psi_{\mathbf{c}}$. The pulses are scaled so that the peak value of the standard pulse is 1 . Note how the proposed method controls the RF peak power over all four waveforms.

Slices 1 and 3 are shifted by FOV/2. Both the standard as well as the proposed pulses give accurate shifts without leakage artifacts (18).

\section{DISCUSSION}

As shown in previous work (15), the phase relaxation for multiband excitation can effectively reduce the peak amplitude of the RF pulse. The aim of this work was to cast the phase relaxation problem into a generalized and robust procedure that allows for (1) combination with the CAIPIRINHA phase cycling scheme and (2) extremely short computation time. Note that the peak reduction is obtained by relaxing the phase of the
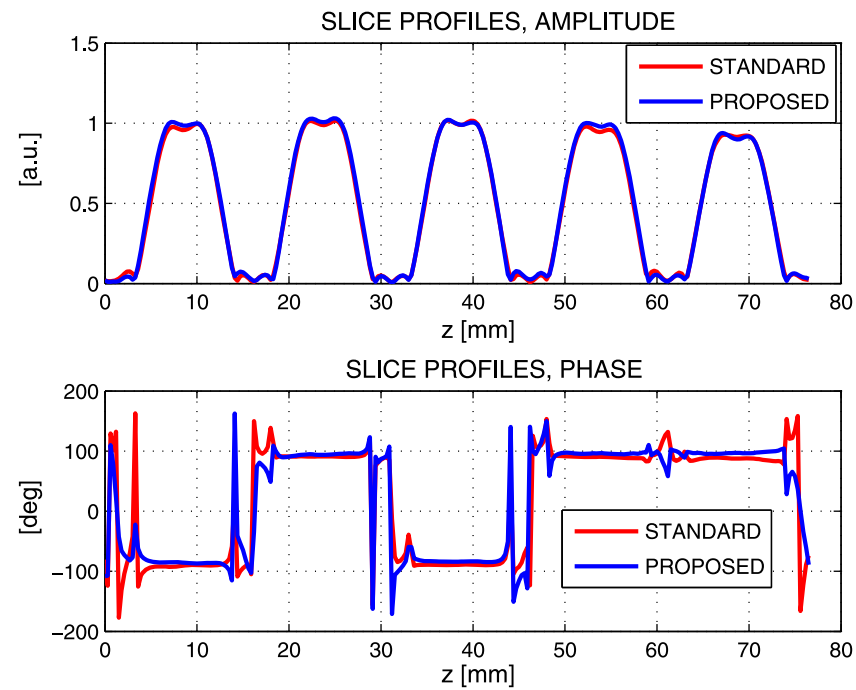

Figure 6. Experiment 3: scanner implementation. Amplitude and phase

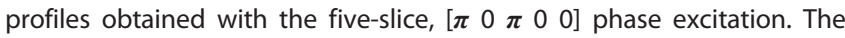
phase plots are shifted by $90^{\circ}$ to avoid phase jumps around $\pm 180^{\circ}$. The phase obtained from the $\left[\begin{array}{llll}0 & 0 & 0 & 0\end{array}\right]$ is subtracted from the datasets. The plots refer to the images obtained with the standard (no phase relaxation) and proposed pulses, as calculated in Experiment 2. Note the retained accuracy of the excitation, obtained with drastically reduced RF peak power (see also Experiment 2).

\section{slice $1 \quad$ slice 2 slice $3 \quad$ slice 4 slice 5}

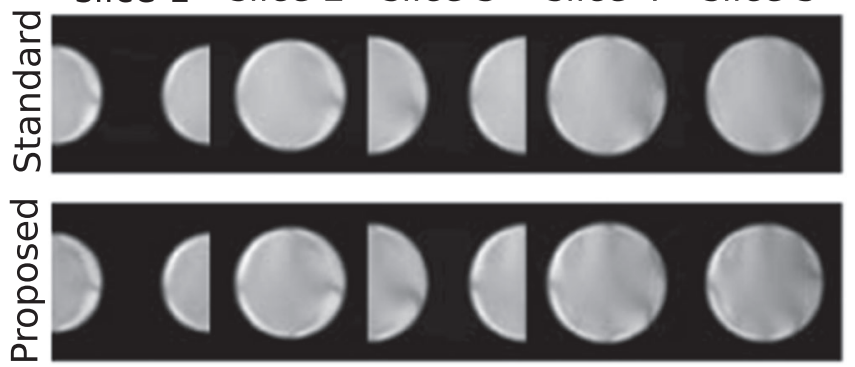

Figure 7. Experiment 3: scanner implementation of alternating pulses. The images corresponding to Slices 1 and 3 are accurately shifted by FOV/2 in both cases. This proves the practical applicability of the proposed pulses, which exhibit drastically reduced RF peak power with respect to the standard pulses.

slices. In contrast to (11) and (12), the length of the pulses does not change.

The proposed method shows better convergence properties than the method from (15), as illustrated in Experiment 1. This improvement is related to the smooth form of the objective function and constraints in Equations A9-A11, derived by introducing the auxiliary variable $t$, which makes it possible to cast the problem as a linear program with quadratic constraints. Since the gradients of the corresponding objective and constraint functions are explicitly known, the minimization can be quickly performed by an active-set algorithm. The convergence test (Fig. 3) shows that the proposed method is less sensitive to local minima, that is, it has a much higher chance to converge to the global optimum than the implementation based on (15).

The extension of the phase relaxation approach to the CAIPIRINHA technique is shown to be relevant in the second experiment. The proposed method can deal with any phase cycling scheme to minimize the overall peak amplitude. The optimal interslice phase depends on the CAIPIRINHA scheme, which covers a large range of possibilities, in number of slices, $N$, and the corresponding desired shifts, as recently shown in (17). For these reasons, a look-up table approach such as the one introduced in (15) is no longer feasible, and the interslice phase calculation should be computed online. The method proposed offers 
a solution to this problem and the authors provide the MATLAB implementation, hoping that it will be a practical aid for the application of the CAIPIRINHA technique.

Performing a complete benchmark of the two phase relaxation methods in terms of computation time is not straightforward, given the many factors that influence the computation time. These are the number of restarts, the number of iterations for each restart, the number of sample points in the pulse etc. We implemented the "brute-force" optimization from (15) for the CAIPIRINHA setups, and we found that a run with 1000 restarts could require minutes. The ability of the new formulation to exploit the structure of the mathematical problem results in an elegant and fast algorithm that can achieve global convergence in about $1 \mathrm{~s}$.

As mentioned in the theory section, the proposed method can be easily adapted to pTx systems $(19,20)$, when phase and amplitude shimming sets are channel and slice dependent. Given $P$ transmitting channels, denote by $\psi_{p, n}$ the phase shimming for channel $p$ and slice $n$, and by $\alpha_{p, n}$ the corresponding amplitude weight. It follows that the $p$ th channel waveform will be $f^{p}(t, \vec{\phi})=\sum_{n=1}^{N} b(t) \alpha_{p, n} \mathrm{e}^{\mathrm{i}\left(\gamma G t z_{n}+\psi_{p, n}+\phi_{n}\right)} \quad$ and the solution to the peak minimization problem (Equation [6]) can be obtained with the proposed method by including the weights $\alpha$ in the matrices $Q_{i}$. Our MATLAB code includes this option.

Expansion to pTx for multi-band CAIPIRINHA is straightforward and can benefit from the compact algorithm introduced here. Furthermore, the interslice phase relaxation could be useful to reduce peak power in PTx spoke pulses (21), and the procedure described in this work can be adapted for this particular case.

A final remark on the total power used: it is simple to show that the interslice phase relaxation has no effect on the total power of the multi-band RF pulses for standard (no pTx) setups. However, the phase relaxation could be exploited to minimize the global SAR of the pTx pulses. The methodology illustrated in the present work could be combined with more comprehensive approaches, for instance (22). Given the speed of computations and good convergence performance, our method is expected to be easily included in this type of large scale optimization strategy, where interslice phases depend on the channel and possibly on the spoke. The number of variables would then be larger $\left(N \times N_{\mathrm{c}} \times N_{\mathrm{s}}\right.$, where $N_{\mathrm{c}}$ and $N_{\mathrm{s}}$ represent, respectively, the number of pTx channels and the number of spokes) and the quick convergence resulting from our formulation should benefit the design step. A study in this direction goes beyond the scope of this work but it could be investigated in the future.

\section{CONCLUSION}

The minimum RF peak amplitude problem with phase relaxation is implemented as a linear program with quadratic constraints. The generalized approach also includes the possibility to design minimum peak amplitude RF pulses for CAIPIRINHA phase cycling schemes. Simulations show that the optimal interslice phase settings are scan dependent and thus they should be computed online. We provide a MATLAB implementation that makes this possible. The improved performance with respect to the standard multi-slice pulses and the existing interslice pulses is shown. Experiments on a 7T scanner confirm the validity and demonstrate the practical feasibility of the proposed low RF peak approach.

\section{Acknowledgements}

The research leading to these results has received funding under a grant agreement from the Holides project no 332933 of the ARTEMIS Joint Undertaking.

MRI data acquisition was in part covered by the Scannexus/ Brains Unlimited intramural development grant D0111.

\section{REFERENCES}

1. Souza SP, Szumowski J, Dumoulin CL, Plewes DP, Glover G. SIMA: simultaneous multislice acquisition of MR images by Hadamardencoded excitation. J. Comput. Assist. Tomogr. 1988; 12: 1026-1030.

2. Müller, S. Multifrequency selective if pulses for multislice MR imaging. Magn. Reson. Med. 1988; 6: 364-371.

3. Glover GH. Phase-offset multiplanar (POMP) volume imaging: a new technique. J. Magn. Reson. Imaging 1991; 1: 457-461.

4. Larkman DJ, Hajnal JV, Herlihy AH, Coutts GA, Young IR, Ehnholm G. Use of multicoil arrays for separation of signal from multiple slices simultaneously excited. J. Magn. Reson. Imaging 2001; 13: 313-317.

5. Moeller S, Yacoub E, Olman CA, Auerbach E, Strupp J, Harel N, Ugurbil K. Multiband multislice GE-EPI at 7 Tesla, with 16-fold acceleration using partial parallel imaging with application to high spatial and temporal whole-brain FMRI. Magn. Reson. Med. 2010; 63: 1144-1153.

6. Feinberg DA, Moeller S, Smith SM, Auerbach E, Ramanna S, Glasser MF, Miller KL, Ugurbil K, Yacoub E. Multiplexed echo planar imaging for sub-second whole brain FMRI and fast diffusion imaging. PLoS One 2010; 5: e15710.

7. Breuer FA, Blaimer M, Heidemann RM, Mueller MF, Griswold MA, Jakob PM. Controlled aliasing in parallel imaging results in higher acceleration (CAIPIRINHA) for multi-slice imaging. Magn. Reson. Med. 2005; 53: 684-691.

8. Pruessmann KP, Weiger $M$, Scheidegger MB, Boesiger P. SENSE: sensitivity encoding for fast MRI. Magn. Reson. Med. 1999; 42(5): 952-962.

9. Setsompop K, Gagoski BA, Polimeni JR, Witzel T, Wedeen VJ, Wald LL. Blipped-controlled aliasing in parallel imaging for simultaneous multislice echo planar imaging with reduced $g$-factor penalty. Magn. Reson. Med. 2012; 67: 1210-1224. DOI: 10.1002/mrm.23097

10. Maudsley AA. Multiple-line-scanning spin-density imaging. J. Magn. Reson. 1980; 41: 112-126.

11. Conolly SM, Nishimura DG, Macovski A. Variable-rate selective excitation. J. Magn. Reson. 1988; 78: 440-458.

12. Auerbach EJ, Xu J, Yacoub E, Moeller S, Ugurbil K. Multiband accelerated spin-echo echo planar imaging with reduced peak RF power using time-shifted RF pulses. Magn. Reson. Med. 2013; 69: $1261-1267$.

13. Norris DG, Koopmans PJ, Boyacioglu R, Barth M. Power independent of number of slices (PINS) radiofrequency pulses for low-power simultaneous multislice excitation. Magn. Reson. Med. 2011; 66: 1234-1240.

14. Hennig J. Chemical shift imaging with phase-encoding RF pulses. Magn. Reson. Med. 1992; 25(2): 289-998.

15. Wong E. Optimized phase schedules for minimizing peak RF power in simultaneous multi-slice RF excitation pulses. In Proceedings of the 20th Annual Meeting of ISMRM, Melbourne, Australia, 2012. p. 2209.

16. Hecht E. Optics, 2nd edn. Addison-Wesley, Berkeley, CA USA, 1987, p. 246.

17. Stemkens B, Tijssen RH, Andreychenko A, Crijns SPM, Sbrizzi A, Lagendijk JJW, van den Berg CAT. Optimizing CAIPIRINHA multiband acquisition scheme for $2 \mathrm{D}$ multi-slice experiments in the abdomen. In Proceedings of the 22nd Annual Meeting of ISMRM, Milano, Italy, 2014. p. 645.

18. Xu J, Moeller S, Auerbach EJ, Strupp J, Smith SM, Feinberg DA, Yacoub E, Ugurbil K. Evaluation of slice accelerations using multiband echo planar imaging at 3 T. Neuroimage 2013; 83: 991-1001.

19. Wu X, Schmitter S, Auerbach EJ, Moeller S, Ugurbil K, Van de Moortele P-F. Simultaneous multislice multiband parallel radiofrequency excitation with independent slice-specific transmit B1 homogenization. Magn. Reson. Med. 2013; 70: 630-638.

20. Poser BA, Anderson RJ, Guérin B, Setsompop K, Deng W, Mareyam A, Serano P, Wald LL, Stenger VA. Simultaneous multislice excitation by 
parallel transmission. Magn. Reson. Med. 2014; 71: 1416-1427. DOI: 10.1002/mrm.24791

21. Saekho S, Yip CY, Noll DC, Boada FE, Stenger VA. Fast- ${ }^{k-z}$ threedimensional tailored radiofrequency pulse for reduced $B-1$ inhomogeneity. Magn. Reson. Med. 2006; 55(4): 719-724.

22. Guerin B, Setsompop K, Ye H, Poser BA, Stenger AV, Wald LL. Design of parallel transmission pulses for simultaneous multislice with explicit control for peak power and local specific absorption rate. Magn. Reson. Med. 2015; 73: 1946-1953. DOI: 10.1002/mrm.25325

23. Boyd S, Vandenberghe L. Convex Optimization. Cambridge University Press, 2004.

\section{Appendix A: Mathematical derivation}

We will now derive Equation [6]. The discretized version of the single-band pulse $b(t)$ is denoted by the column vector $\boldsymbol{b}$. We define the matrix $\boldsymbol{B}$ as the horizontal concatenation of $\boldsymbol{b}$ weighted by the linear phase terms $\mathrm{e}^{\mathrm{i} \gamma G t z_{n}}$ such that $\boldsymbol{B}_{h, n}=b\left(h \Delta_{t}\right) \mathrm{e}^{\mathrm{i} \gamma G h \Delta_{t} z_{n}}$, where the index $h$ runs from 1 to $T / \Delta_{t}, \Delta_{t}$ is the discretization time step and $T$ the length of the pulse $b$. By defining the intraslice phase vector $\boldsymbol{u}$ as $\boldsymbol{u}_{n} \equiv \mathrm{e}^{\mathrm{i} \phi_{n}}$, the (discretized) $N$-band excitation RF pulse from Equation [2] is simply given by $\mathbf{B u}$.

The CAIPIRINHA phase cycling scheme can be easily implemented in this matrix-vector model in the following way. Given a scheme $\Psi$, construct the matrix $\boldsymbol{A}$ such that

$$
A \equiv\left[\begin{array}{cccc}
\mathrm{e}^{\mathrm{i} \psi_{1,1} \boldsymbol{B}(:, 1)} & \mathrm{e}^{\mathrm{i} \psi_{1,2} \boldsymbol{B}(:, 2)} & \ldots & \mathrm{e}^{\mathrm{i} \psi_{1, N} \boldsymbol{B}(:, N)} \\
\mathrm{e}^{\mathrm{i} \psi_{2,1} \boldsymbol{B}(:, 1)} & \mathrm{e}^{\mathrm{i} \psi_{2,2} \boldsymbol{B}(:, 2)} & \ldots & \mathrm{e}^{\mathrm{i} \psi_{2, N} \boldsymbol{B}(:, N)} \\
\vdots & \vdots & \ddots & \vdots \\
\mathrm{e}^{\mathrm{i} \psi_{P, 1} \boldsymbol{B}(:, 1)} & \mathrm{e}^{\mathrm{i} \psi_{P, 2} \boldsymbol{B}(:, 2)} & \ldots & \mathrm{e}^{\mathrm{i} \psi_{P, N} \boldsymbol{B}(:, N)}
\end{array}\right]
$$

In the previous equation, we made use of the MATLAB notation $\boldsymbol{B}(:, n)$ indicating the $n$th column of $\boldsymbol{B}$. Clearly, the product $\boldsymbol{A} \boldsymbol{u}$ gives the vertical concatenation of all $P$ pulses needed for the cycling scheme $\Psi$.

The generalized minimum peak RF multi-band/CAIPIRINHA problem becomes $^{1}$

$$
\begin{aligned}
& \text { minimize }\|\boldsymbol{A} \boldsymbol{u}\|_{\infty} \\
& \text { such that }\left|u_{n}\right|=1 \text { for } n=1, \ldots, N .
\end{aligned}
$$

Introducing the auxiliary variable $t$ (23) and defining ${ }^{2}$ $\mathbf{z} \equiv\left[\mathfrak{R}\left(\boldsymbol{u}^{\top}\right) \mathfrak{I}\left(\boldsymbol{u}^{\top}\right)\right]^{\top}$, the problem from Equation [A14] can be written in the equivalent form:

$$
\text { minimize } t
$$

$$
\begin{gathered}
\text { such that }\left\|\left[\begin{array}{cc}
\mathfrak{R}\left(a_{h}^{\top}\right) & -\mathfrak{I}\left(a_{h}^{\top}\right) \\
\mathfrak{I}\left(a_{h}^{\top}\right) & \Re\left(a_{h}^{\top}\right)
\end{array}\right]\right\|_{2} \mathbf{z} \leq t, \quad \forall h \\
{\left[\begin{array}{ll}
z_{n} & z_{n+N}
\end{array}\right]\left[\begin{array}{c}
z_{n} \\
z_{n+N}
\end{array}\right]=1, \quad n=1, \ldots, N .}
\end{gathered}
$$

In Equation [A4], $a_{h}$ refers to the $h$ th row of the matrix $\boldsymbol{A}$ and the index $h$ goes over all rows, namely, over all RF sample points. Note that $t \in \mathbb{R}$ and $\boldsymbol{z} \in \mathbb{R}^{2 N}$, that is, all variables are real. Furthermore, the $\|\cdot\|_{\infty}$ norm is substituted by the Euclidean norm.

\footnotetext{
'The $\ell_{\infty}$ norm on $\mathbb{C}^{p}$ is defined as $\|\boldsymbol{v}\|_{\infty}=\max _{i=1, \ldots, p}\left|v_{i}\right|$.

${ }^{2} \mathfrak{R}(\cdot)$ and $\mathfrak{\Im}(\cdot)$ are, respectively, the real and imaginary parts of a complex array.
}

Finally, we introduce the vectors $\boldsymbol{y}$ and $\boldsymbol{c}$, both of length $2 N+1$ and defined as $\boldsymbol{y} \equiv[\mathbf{z} ; t]$ and $\boldsymbol{c} \equiv[0, \ldots, 0,1]^{\top}$, and the matrices $\boldsymbol{Q}_{h}$ defined as

$$
\boldsymbol{Q}_{h} \equiv\left[\begin{array}{cc}
\mathfrak{R}\left(a_{h}^{\top}\right) & -\mathfrak{I}\left(a_{h}^{\top}\right) \\
\mathfrak{I}\left(a_{h}^{\top}\right) & \mathfrak{R}\left(a_{h}^{\top}\right)
\end{array}\right] .
$$

The optimization problem becomes

$$
\text { minimize } \boldsymbol{c}^{\top} \boldsymbol{y}
$$

$$
\begin{gathered}
\text { such that } \boldsymbol{y}^{\top}\left[\begin{array}{cc}
\boldsymbol{Q}_{h}^{\top} \boldsymbol{Q}_{h} & 0 \\
0 & -1
\end{array}\right] \boldsymbol{y} \leq 0, \quad \forall h \\
{\left[\begin{array}{ll}
y_{n} & y_{n+N}
\end{array}\right]\left[\begin{array}{c}
y_{n} \\
y_{n+N}
\end{array}\right]=1, \quad n=1, \ldots, N .}
\end{gathered}
$$

The equality constraint from Equation [A8] can be written in the more compact form $\boldsymbol{y}^{\top} \boldsymbol{P}_{n}^{\top} \boldsymbol{P}_{n} \boldsymbol{y}-1=0$, where $\boldsymbol{P}_{n}$ is the $2 \times(2 N+1)$ matrix of zeros and ones defined as

$$
\boldsymbol{P}_{n}(i, j)= \begin{cases}1 & (i, j)=(1, n) \\ 1 & (i, j)=(2, n+N) \\ 0 & \text { otherwise }\end{cases}
$$

In conclusion, denoting the matrix of the inequality constraints as $Q_{h}$ and setting $P_{n} \equiv \boldsymbol{P}_{n}^{\top} \boldsymbol{P}_{n}$, we have the final version of the minimization problem:

$$
\begin{array}{ll}
\text { minimize } & \boldsymbol{c}^{\top} \boldsymbol{y} \\
\text { such that } & \boldsymbol{y}^{\top} Q_{h} \boldsymbol{y} \leq 0, \quad \forall h \\
& \boldsymbol{y}^{\top} P_{n} \boldsymbol{y}-1=0, \quad n=1, \ldots, N
\end{array}
$$

which is a linear program with quadratic equality and inequality constraints.

\section{Appendix B: Matlab functions}

The main function optim_phi.m and the two subroutines fun.m and nonlinconstr.m are listed below. As an example, the input values for Experiment $2\left(\Psi_{\mathrm{a}}\right.$ from Equation (3)) are also given below.

The Matlab minimization function fmincon is needed.

Main function optim_phi.m

function [phi peak] =optim_phi(Psi, b, dt, G, z, alpha) $\%$ This code computes optimal interslice phases for MB-pTx \% INPUT:

$\%$ Psi : the excitation phases. Psi $(p, n)$ is the phase of pulse $p$ and slice $n$, in [rad]

$\%$ b : the discretized single band pulse, column vector $\% d t \quad:$ the discretization time step

$\%$ : : the value of the slice selective gradient, in $[\mathrm{G} / \mathrm{cm}]$ $\% \mathrm{z} \quad$ : the coordinates of the slice centers, row vector in $[\mathrm{cm}]$ \%alpha : (optional) the amplitude shimming settings, same format as Psi 
\% OUTPU:

$\%$ phi : the optimal interslice phases for each slice, in [rad] $\%$ peak : the RF peak value obtained with the optimized phases $\%$

\% by Alessandro Sbrizzi, December 2014.

$\%$ REFERENCE: ......

$\%$

\% contact: a.sbrizzi@umcutrecht.nl

$\%$ initialize

garma $=26753.0 ; \%$ [Gauss, cm, Hertz]

Nt = length $(b)$;

theta $=$ gamma* $\mathrm{G}^{*} \mathrm{z}$;

$t=(0: d t:(N t-1) * d t) .^{\prime} ;$

$[\mathrm{P} \mathrm{N}]=\operatorname{size}(\mathrm{Psi})$;

if nargin $==5$ end alpha = Psi.^^; \% set amplitude settings $=1$

$a=$ alpha. ${ }^{\wedge} \exp \left(1 i^{*}\right.$ Psi $)$;

$\%$ Problem matrix

$\mathrm{A}=[]$;

for $p=1: P$

$\mathrm{F}=\operatorname{zeros}(\mathrm{Nt}, \mathrm{N})$;

for $\mathrm{j}=1: \mathrm{N}$ end$$
F(:, j)=a(p, j) * b . * \exp (1 i * \operatorname{theta}(j) * t) ;
$$

end

$$
A=[A ; F] \text {; }
$$

\section{$\%$ INITIALIZE OPTIMIZATION}

$\mathrm{N}$ restarts $=5$;

c = $\left[\right.$ zeros $\left.\left(2^{*} \operatorname{size}(A, 2), 1\right) ; 1\right]$;

$\%$ construct inequality and equality constr matrices

a2 = 2*size $(A, 2)+1$;

Aineq $=$ zeros $(\operatorname{size}(A, 1) * a 2, a 2)$;

Aeq $=\operatorname{zeros}(a 2, a 2, \operatorname{size}(A, 2))$;

for $j=1: \operatorname{size}(A, 1)$

matr $=[\operatorname{real}(A(j,:)),-i \operatorname{mag}(A(j,:)) ; \operatorname{imag}(A(j,:))$, $\operatorname{real}(A(j,:))]$;

Aineq $((j-1) * a 2+1: j * a 2-1,1$ :end -1$)=(\text { matr'*matr })^{\prime}$;

end Aineq $(j * a 2$, end $)=-1$;

for $j=1$ :size(Aeq, 3 )

matr = zeros $(2,2 * \operatorname{size}(A, 2))$;

$\operatorname{matr}(1, j)=1$;

$\operatorname{matr}(2, j+\operatorname{size}(A, 2))=1$;

end

Aeq $(1: 2 * \operatorname{size}(A, 2), 1: 2 * \operatorname{size}(A, 2), j)=\operatorname{sparse}($ matr*matr $)$;

n_ineq = size $(A, 1)$;n_eq = size $($ Aeq, 3$)$;

peak = Inf;

options = optimset ('Display' , 'off' , 'MaxIter' ,200,

'GradConstr', 'on', ,...

'GradObj', 'on', 'Algorithm' ,'active-set');

display('OPTIMIZING ...');

randn('state' ,0) ; \% fix seed random generator

rand('state', 0$)$;

starting $=$ rand $\left(\mathrm{N}, \mathrm{N} \_\right.$restarts $) ; \%$ set random starting values $\%$ OPTIMIZE

for $j=1:$ N_restarts

start_y $=\exp (1 i * \operatorname{starting}(:, j) * 2 * p i)$; start_y $=[$ real

(start_y);imag(start_y);1];

[solfval] = fmincon $(@(y)$ fun $(y, c)$, start_y, [] , []$,[],[],[],[], \ldots$

$@(y)$ nonlinconstr (y,Aineq,Aeq, n_ineq, n_eq),

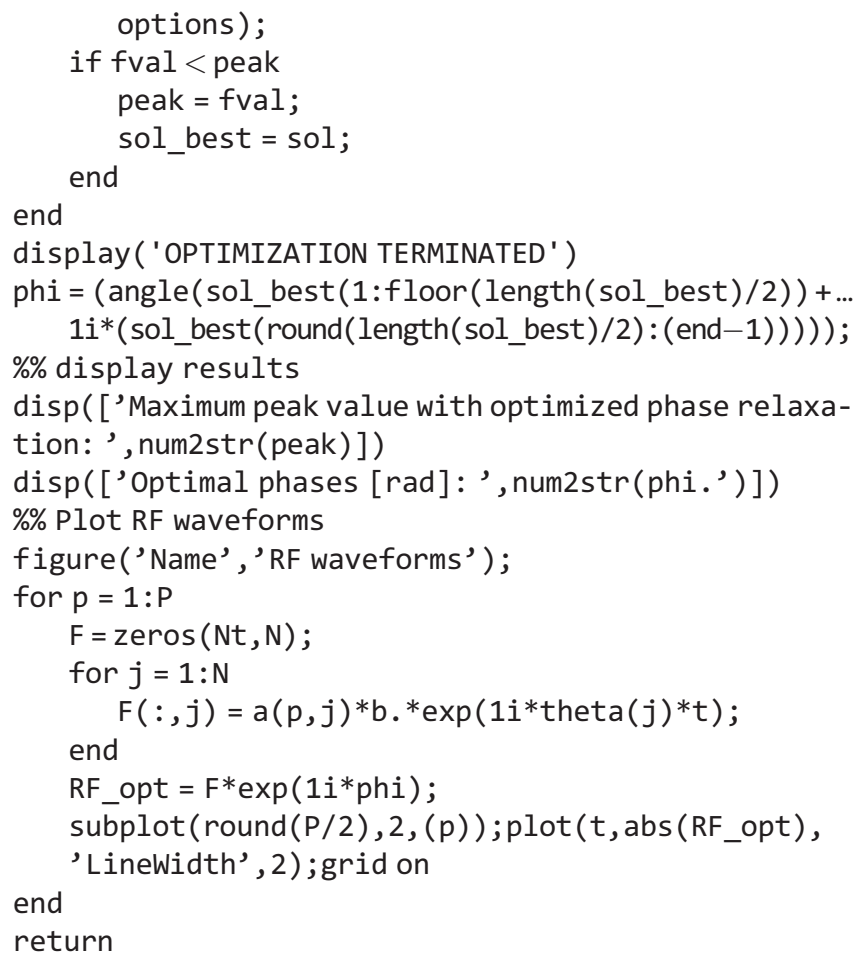

\section{Function fun.m}

function [objgradf] $=$ fun $(y, c)$

obj = transpose (c) ${ }^{*} y$;

ifnargout $>1$

gradf $=\mathrm{C}$;

end

\section{Function nonlinconstr.m}

function $[c, c e q$, gradc, gradceq $]=$ nonlinconstr $(y$, Aineq, Aeq, n_ineq, n_eq)

ceq $=$ zeros $($ n_eq, 1$)$;

for $j=1:$ n_eq

end

$$
\operatorname{ceq}(j)=y^{\prime} * \text { squeeze }(\operatorname{Aeq}(:,:, j)) * y-1 \text {; }
$$

$\mathrm{GC}=2^{*}(\text { Aineq*y })^{\prime} ;$

gradc $=$ reshape $\left(G C\right.$, length $(y), n_{-}$ineq $)$;

$c=0.5^{*}\left(y^{\prime} * \operatorname{gradc}\right)^{\prime} ;$

gradceq $=[]$;

for $j=1: n \_$eq

gradceq $=\left[\right.$ gradceq transpose $\left(2^{*} y^{\prime} * \operatorname{squeeze}(\operatorname{Aeq}(:,:\right.$, j))) ] ;

end

end

\section{Example from Experiment 2}

Psi $=\left[\begin{array}{llllll}0 & 0 & 0 & 0 & 0 ; \text { pi } 0 \text { pi } 0 & 0\end{array}\right]$;

$b=\operatorname{sinc}([-256: 255] / 128) . ;$

$\mathrm{dt}=5.0000 \mathrm{e}-06$;

$\mathrm{G}=1.2 ; \mathrm{z}=\left[\begin{array}{lllll}-4 & -2 & 0 & 2 & 4\end{array}\right]$;

[phi peak] = optim_phi(Psi,b,dt,G,z); 